\title{
LE BUZZ OU L'ART DE PIMENTER LES ÉMISSIONS-DÉBATS
}

\section{RESPONSABILITÉ (ÉNONCIATIVE) DU PRODUCTEUR- PRÉSENTATEUR DANS L'AFFAIRE ZEMMOUR/SY}

\author{
Houda LANDOLSI ${ }^{1}$
}

\begin{abstract}
Cet article s'intéresse aux rôles énonciatifs que joue le producteurprésentateur de l'émission Les Terriens du dimanche, Thierry Ardisson, lors de l'affaire Zemmour/Sy ; une affaire qui éclate suite à un commentaire fait par le polémiste Éric Zemmour sur le prénom de la chroniqueuse Hapsatou Sy. L'objectif de l'article est d'analyser l'articulation entre postures énonciatives et rôles interactionnels du producteur/présentateur de l'émission avant, pendant et après l'affaire. L'étude a pour objectif de montrer que dans son rôle de producteur, Thierry Ardisson agit en tant que sur-énonciateur ; alors que dans son rôle de présentateur/modérateur du débat, il choisit plutôt la posture d'effacement énonciatif, même si cette posture bascule, rapidement, vers une forme de sur-énonciation. L'étude s'arrête également sur le lien sous-jacent entre la responsabilité énonciative du journaliste et sa responsabilité éthique ; un lien explicitement évoqué par des commentateurs de différentes professions et colorations politiques.
\end{abstract}

1 Houda LANDOLSI est docteure en linguistique française au Département des Langues modernes de l'Université d'Uppsala.

Recherches en communication, $\mathrm{n}^{\circ} 53$ - Article publié le 14/01/2022 
Les Terriens du dimanche est une émission de télévision, créée et présentée par Thierry Ardisson et diffusée entre septembre 2017 et avril 2019 sur la chaîne française privée C8. L'émission a eu deux saisons durant lesquelles elle a connu quelques changements, mais le concept reste fondamentalement le même : retour sur les événements marquants de la semaine (ou du mois) par les chroniqueurs, mais aussi par un invité qui est au cour de l'actualité. Les Terriens du dimanche peut être catégorisée comme une émission-débat (talk-show) et elle a fait parler d'elle entre autres suite à deux polémiques qui ont fait perdre à l'équipe deux de ses chroniqueurs : l'affaire dite du « JeremstarGate » et l'affaire Zemmour/Sy.

L'affaire Zemmour/Sy éclate lors de l'enregistrement de l'épisode du 16 septembre 20182. Thierry Ardisson (désormais TA) reçoit Éric Zemmour (ÉZ) qui fait la promotion de son nouveau livre Destin français. Interrogé sur sa réaction déplorant le choix de Rachida Dati de prénommer sa fille Zohra, ÉZ affirme regretter, encore et toujours, l'abolition de la loi imposant aux parents de donner à leur enfant un prénom du calendrier des saints. Hapsatou Sy (HS), une chroniqueuse de l'émission, rappelle à l'invité qu'elle s'appelle précisément... Hapsatou, ce à quoi ÉZ répond : votre prénom [...] est une insulte à la France $^{3}$.

Dans la séquence diffusée de l'échange, TA n'a pas explicitement pris parti dans la polémique. Il s'est ensuite exprimé, précisant, en son nom et au nom des producteurs, le positionnement des réalisateurs et de la chaîne, donnant sa perception des faits, expliquant ses choix et commentant, surtout, la réaction d'HS, qu'il trouve excessive. L'affaire a abouti à un accord postulant qu'HS quitte l'émission.

Les trois protagonistes (et même les chroniqueurs de l'émission) ont tous eu l'occasion de revenir sur cette affaire, proposant ainsi leur version des faits et jouissant d'une sorte de droit de réponse $e^{4}$. Mais c'est

2 «L'affaire Hapsatou Sy - Éric Zemmour - Les Terriens du Dimanche - 16/09/2018», Les Terriens, 01/10/2018, $11 \mathrm{~min} 36 \mathrm{~s}$. https://www.youtube.com/watch? $=x F Z e-$ hICfmNk.

3 « Hapsatou Sy insultée par Éric Zemmour », HapsatouSy, 18/09/2018, 5min08s. https://www.youtube.com/watch? $\mathrm{v}=\mathrm{wIBB} 231 \mathrm{JK} 3 \mathrm{o}$ (dernière consultation le 30/01/2021). Nouveau lien : https://www.dailymotion.com/video/x6u113d.

4 Hapsatou Sy : "Je demande des excuses à Éric Zemmour », Touche pas à mon poste !, 27/09/2018, 23min00s. https://www.youtube.com/watch?v=uyG9S2xuvyE. Éric Zemmour : « Menacé de plainte, Éric Zemmour dénonce "le règne de l'émotion et de la victimisation" ", BFMTV, 17/09/2018, 4min55s. https://www.youtube.com/ 
surtout HS qui s'est exprimée à plusieurs reprises sur ce qu'elle avait considéré comme une des pires expériences qu'elle avait dû endurer. Dans le cadre restreint de cette étude, nous n'aurons malheureusement pas l'occasion de revenir sur les positionnements par redoublement autodialogique $^{5}$ de tous les protagonistes de l'histoire. Notre intérêt portera, surtout, sur le positionnement et la responsabilité énonciative des producteurs de l'émission, incarnés dans la personne de TA, visà-vis de l'échange polémique, lors de l'enregistrement de l'émission, mais aussi avant et après sa diffusion, et à la suite du buzz que l'épisode a créé. Même si notre intérêt portera, principalement, sur la responsabilité énonciative, celle-ci implique, nécessairement, une responsabilité juridique (qui sera évoquée par les producteurs eux-mêmes) et une responsabilité morale et éthique.

\section{Retour sur l'échange polémique}

L'affaire Zemmour/Sy apparaît sous la forme de deux séquences : une séquence diffusée par la chaîne $\mathrm{C} 8$ avec montage et une autre mise en ligne par HS sans montage.

Voici la transcription de ce que la chaîne a diffusé :

1.

ÉZ elle [rachida dati] est ministre de la république française/ elle est censée être française/ (.) même si elle a la double nationalité/ je suis contre la double nationalité avec le maroc/ (.) et que : (.) normalement euh chez moi/ (.) en tout cas depuis : (.) une loi de bonaparte qui a malheureusement été abolie en mille neuf cent quatre-vingt-treize par/ (.) les socialistes/ (.) euh on doit donner des- des prénoms/ (.) dans ce qu'on appelle le calendrier $\backslash$ (.) c'est-à-dire/ (.) les saints chrétiens $\backslash$

VoIx oui/ mais enfin bon $\backslash$

watch? $=87 \mathrm{xggNQN4zg \& t=2s.} \mathrm{Thierry} \mathrm{Ardisson} \mathrm{:} \mathrm{«} \mathrm{Thierry} \mathrm{Ardisson} \mathrm{agacé} \mathrm{par}$ Hapsatou Sy : il parle », Actu Médias, 18/09/2018, 6min21s. https://www.youtube. $\mathrm{com} /$ watch? $\mathrm{v}=\mathrm{y} 99 \mathrm{bkK} 4 \mathrm{~s} 2 \mathrm{Og} \& \mathrm{t}=63 \mathrm{~s}$ (dernière consulation le 30/01/2021). Nouveau lien: https://www.lexpress.fr/actualite/medias/zemmour-chez-ardisson-hapsatousy-sur-la-sellette_2035701.html.

5 Par redoublement autodialogique (Rabatel, 2012, pp. 31-32), nous désignons la nouvelle position énonciative qu'adopte le même énonciateur par rapport à son discours antérieur. 
HS alors moi je m'appelle hapsatou hein/ ((rires)) [XXX]

ÉZ [eh ben eh ben] eh ben eh ben votre mère a eu tort

HS ah bon $/ /$ ma mère $\mathbf{a} / /=$

ÉZ $\quad=$ a eu tort

HS elle- elle a eu tort//

ÉZ ah oui/

HS vous voudriez qu'elle m'appelle : [m- marine]

ÉZ [oui]

HS = ou des prénoms qui/ (.) ne lui inspirent/

HS [absolument rien//]

ÉZ [absolument/ c'est exactement ce que je veux//]

ÉZ [c'est exactement ce que je veux//]

HS vous voudriez donc que je m'appelle comment//

ÉZ = corinne/ (.) ça vous irait très bien\

HS vous trouvez que corinne ça serait pas mal $\backslash$

ÉZ ça serait parfait।

HS ce type est incroyable $\backslash$

((rires : public et TA))

$((\text { applaudissements }))^{6}$

Selon ÉZ, la mère d'HS a bien eu tort de lui donner ce prénom. L'énoncé porte un jugement de valeur négatif à la fois sur le prénom de la chroniqueuse et sur les capacités de jugement de sa mère (avoir tort).

Dans la séquence sans montage, ÉZ va encore plus loin :

2.

HS vous allez- vous allez entendre ce que j'ai à vous dire\

ÉZ oui moi aussi XXX

HS jamais/ (.) je n'ai entendu quelque chose d'aussi blessant $\backslash$ (.) parce que pour moi qui aime m- ma france/ (.) et qui aime ce pays que ça vous plaise ou que ça vous déplaise/ (.) je trouve que ce que vous venez de dire/ (.) n'est pas une insulte à mon égard/ (.) c'est une insulte/ (.) à la francel

6 «L'affaire Hapsatou Sy - Éric Zemmour - Les Terriens du Dimanche - 16/09/2018», Les Terriens, 01/10/2018, $11 \mathrm{~min} 36 \mathrm{~s}$. https://www.youtube.com/watch? $\mathrm{v}=\mathrm{xFZehl-}$ CfmNk. 
((applaudissements))

ÉZ [mademoiselle]

((applaudissements))

ÉZ [mademoiselle]/ (.) c'est votre prénom qui est une insulte à la france/

HS [mon prénom est une insulte à la france//]

ÉZ [parce que la france] la france n'est pas/ (.) une terre vierge/

HS [mon prénom est une insulte à la france//]

ÉZ [je vous ai laissé parler/] la france n'est une terre vierge/

HS [mais je XXX]

ÉZ la france n'est pas une terre vierge/ c'est une contrée avec une histoire/ (.)

HS oui//

ÉZ avec une histoire/ avec un passé/

HS d'accord/

ÉZ et les prénoms incarnent/ (.) l'histoire de la france/

HS d'accord/

ÉZ donc/ (.) votre prénom n'est PAS de l'histoire de la france/ (.) que ça vous plaise ou non\

HS mais je suis dans l'histoire de la france/

ÉZ non pas du tout/ (.) vous êtes dans le présent de la france/ mais pas dans l'histoire de la francel ${ }^{7}$

Le recours au terme insulte pour qualifier un prénom est en soi une insulte, aux yeux d'HS, qui exprime sa consternation par le recours, à deux reprises, à la question mon prénom est une insulte à la france/ $/ 8$.

7 « Hapsatou Sy insultée par Éric Zemmour », HapsatouSy, 18/09/2018, 5min08s. https://www.youtube.com/watch? $\mathrm{v}=\mathrm{wIBB} 231 \mathrm{JK} 3 \mathrm{o}$ (dernière consultation le 30/01/2021). Nouveau lien : https://www.dailymotion.com/video/x6u113.

8 Invitée par Cyril Hanouna, HS revient sur les sentiments et émotions qu'elle a dû ressentir lors de l'enregistrement de l'épisode des Terriens du dimanche. Elle emploie, entre autres, les termes : humilier (et humiliée), insulter, consternés, (séquence) douloureuse. "Hapsatou Sy: "Je demande des excuses à Éric Zemmour" ", Touche pas à mon poste!, 27/09/2018, 23min0s. https://www.youtube. $\mathrm{com} / \mathrm{watch}$ ?v=uyG9S2xuvyE \&t=160s. 


\section{Corpus et contexte de l'étude}

La présente étude fait partie d'un projet qui s'intéresse aux représentations qu'ont à la fois les partis politiques et les individus sur l'intégration d'une minorité particulière, à savoir la minorité arabo-musulmane, dans deux contextes géographiques et socio-historiques différents, en l'occurrence en France et en Suède'.

Ne s'intéressant qu'au volet français de l'étude intégrale, le présent article est basé sur un corpus composé de textes français oraux ${ }^{10}$ mis en ligne sur YouTube entre le 17 décembre 2010 (début du printemps arabe) et le 25 janvier 2020 (trois mois après la date du bilan de mi-mandat d'Emmanuel Macron). Le corpus est composé de 950 textes et d'un nombre total de 455745 mots $^{11}$.

L'intégration par le prénom est une question qui est posée dans les médias français, même si cette question n'apparaît pas comme un sujet qui suscite l'intérêt des politiciens durant les campagnes électorales ou même durant d'autres périodes. Selon les données de notre corpus, Marine Le Pen serait la seule candidate à la présidentielle qui s'est prononcée sur la question de la francisation des prénoms et elle prône une assimilation par le prénom ${ }^{12}$. Le Pen parle d'assimilation des immigrés, plutôt que d'intégration. Les deux termes ne sont pas synonymes certes et ne renvoient pas à une même réalité. De même, les objectifs de l'assimilation ne sont pas ceux de l'intégration.

Dans son acception sociologique, l'assimilation est un processus (et non pas un état) durant lequel l'immigré adopte les particularités de la société d'accueil et accepte de les faire siens. Il doit donc renoncer à sa première appartenance et s'approprier la langue, la culture, les normes et le mode de vie des autochtones. L'assimilation est le processus de se forger une nouvelle identité ${ }^{13}$.

9 Le projet est financé par The Swedish Research Council.

10 Les normes et conventions de transcription que nous avons adoptées ont été établies par le groupe ICOR (CNRS - Lyon 2 - ENS de Lyon). Pour d'amples informations sur ces normes, voir http://icar.cnrs.fr/projets/corinte/documents/2013_Conv_ ICOR_250313.pdf.

11 Dans le cadre, restreint, de cet article, il nous est matériellement impossible de décrire les critères de choix et de délimitation du corpus.

12 « Marine Le Pen pour l'assimilation par le prénom français », france aiseetfiere, 30/06/2011, 1 min19s. https://www.youtube.com/watch? $v=0934$ nsi22rk.

13 Voir, pour d'amples informations, Schnapper (2007) et Safi (2006). 
En imposant le terme intégration dans les documents officiaux, la France a choisi, à partir des années 80 , d'abandonner l'assimilation, en tant que concept, mais aussi en tant que politique ${ }^{14}$.

L'immigré n'est plus censé délaisser ses spécificités culturelles, et l'on accepte ou tolère la subsistance de ces particularités au sein de la société d'accueil. L'immigré n'est donc plus obligé de donner à son enfant un prénom français, c'est-à-dire un prénom issu du calendrier des saints. On se rappelle qu'en France, la loi obligeait les parents à sélectionner un prénom du calendrier. La législation était ensuite assouplie le 12 avril 1966 autorisant aux parents de choisir des prénoms régionaux ou issus de la mythologie (Coulmont \& Simon, 2019). Cette restriction n'est levée qu'avec la loi du 8 janvier 1993 qui donne aux parents la possibilité de choisir des noms de baptême originaux (tout en prenant en considération l'intérêt de l'enfant).

Dans l'espace médiatique, c'est surtout le polémiste ÉZ qui se présente comme le fervent défenseur de l'assimilation, entre autres par le prénom. Nous disposons de 24 séquences-vidéo qui portent explicitement et directement sur l'affaire Zemmour/Sy (soit d'un nombre total d'environ 165 minutes de diffusion et de 10264 mots transcrits) $)^{15}$.

\section{Cadres théoriques}

Notre analyse s'inscrit dans la ligne des études effectuées dans le cadre de la linguistique de l'énonciation. Nous n'avons toutefois pas ignoré ce que d'autres disciplines, notamment celle des sciences de l'information et de la communication, offrent. Nous aurons donc recours à certaines notions qui s'inscrivent dans le cadre de ces approches.

Les discours journalistique et médiatique étant situés au carrefour de plusieurs disciplines, nombreux travaux leur sont consacrés. Nous nous contentons d'exposer brièvement quelques études qui peuvent être en lien direct avec le présent article et avec ses notions ${ }^{16}$.

$14 \mathrm{http}: / /$ archives.hci.gouv.fr/-Mots-de-1-integration-.html.

15 Lors d'une ultime dernière relecture, nous avons découvert qu'entre la rédaction de l'article et sa mise en ligne, certains liens ont été modifiés, voire supprimés. Nous avons essayé, dans la mesure du possible, de fournir les nouvelles adresses (dont la source n'est pas nécessairement la plateforme YouTube et par conséquent ne fait pas partie de notre corpus d'étude).

16 Nous avons fait le choix de ne citer qu'une seule et unique définition pour chaque concept-clef. Ce choix s'explique par notre volonté de ne pas encombrer l'analyse 
La notion de modérateur, un rôle qui pourrait qualifier TA, a été l'objet d'étude des travaux de Doury (1995), Hekmat (2020) et Pérennec (2001). Même si ces études portent sur les débats télévisés, la description qu'ils proposent nous semble pertinente pour décrire le rôle énonciatif du présentateur de l'émission Les Terriens du dimanche. Ainsi, et comme le précise Hekmat $(2020, \S 2)$ :

... du point de vue du volume de parole dont [les modérateurs] disposent, ils sont des sous locuteurs, aux plans interactionnel et argumentatif, ils sont en position haute, participant fortement à la structuration, aussi bien interactionnelle que thématique, des échanges, alors qu'au plan énonciatif, leur discours est marqué (comme la plupart des discours journalistiques) par des stratégies d' « effacement énonciatif » qui contribuent à donner l'impression qu'ils sont hors débat.

Nous pouvons remarquer que Hekmat fait la distinction (désormais classique) entre le locuteur, défini comme l'instance première qui produit les énoncés et l'énonciateur, comme la source des points de vue qui s'expriment à travers la prédication de contenus propositionnels dans un énoncé. L'énonciateur correspond donc «à une position (énonciative) qu'adopte le locuteur, dans son discours, pour envisager les faits, les notions, sous tel ou tel point de vue » (Rabatel, 2017, p. 44). En se référant aux objets du discours, l'énonciateur premier se positionne par rapport à eux, en indiquant de quel point de vue (PDV) et dans quel cadre il les envisage.

La question de l'énonciation dans le discours journalistique et médiatique a été évoquée par plusieurs chercheurs (voir, entre autres, Charaudeau, 2006 ; Servais, 2013). La notion large de responsabilité (dans les sciences du langage) a été traitée par Rabatel et Chauvin-Vileno (2006) ; celle de la responsabilité énonciative dans le discours médiatique a été également traitée par Rabatel (notamment 2017). La vaste notion de l'éthique de la télévision a été posée dans plusieurs études, dont l'une des plus récentes est le numéro thématique de la revue Télévision dirigé par Jost \& Pierre et intitulé « Pour une éthique du regard » (2021).

avec de fines distinctions conceptuelles en privilégiant une définition succincte mais précise. 
La responsabilité est d'abord une notion juridique ; elle est aussi éthique et morale ${ }^{17}$. En important cette notion, les sciences du discours et du langage ne l'ont pas dénuée de ses dimensions juridique et éthique. Dans les discours médiatiques, la responsabilité se conçoit et se voit surtout dans l'organisation du texte, la constitution de l'argumentation et de la référence, le choix et la mise en mots de la parole d'autrui ; bref la responsabilité se conçoit dans et à travers le texte : d'où le rapport entre responsabilité et énonciation.

Pour Rabatel et Chauvin-Vileno (2006, $\S \S 30-40)$, la question de responsabilité du sujet parlant a été minimisée, ou en tout cas mise à l'épreuve, par les théories du dialogisme et de la polyphonie de la langue qui ont, à juste titre d'ailleurs, mis l'accent sur l'importance constitutive des relations intersubjectives et interactionnelles dans la construction des énoncés et des discours. Toutefois, le locuteur/énonciateur premier (L1/E1) reste responsable du PDV de l'ensemble de son discours. Nous adoptons, dans ce qui suit, la définition de la responsabilité en linguistique que propose Rabatel (2017, p. 137) :

Ce n'est plus seulement ce que le locuteur assume, c'est aussi le choix des expressions explicites ou implicites de manières de voir, de penser à partir desquelles la réalité est représentée en discours, le choix de ce que L1/E1 décide de mettre hors cadre, le choix des cadres notionnels à partir desquels L1/E1 rend compte des évènements, présente les acteurs.

\section{Ce qui ne peut pas être diffusé peut-il être dit ? Origine de la polémique}

Affirmant avoir été indignée par les propos d'ÉZ, HS poste, sur sa chaîne YouTube, la séquence sans montage, " sous un coup de tête » (sans avoir demandé/eu l'accord des producteurs). En postant la séquence sans montage, HS décrit les propos d'ÉZ comme blessants et violents, mais n'accuse pas directement l'invité de racisme, même si le terme apparaît dans son discours commentant et justifiant sa décision de révéler au grand public la séquence coupée :

17 Pour une brève histoire de la notion de responsabilité, voir, entre autres études, Rabatel et Chauvin-Vileno (2006). 
3.

HS

personnellement et dans mon être// (.) je ne peux PAS accepter/ (.) que/ éric zemmour/ (.) soit parti du plateau/ (.) en ricanant /(.) en me regardant/ (.) impunément/ (.) comme si/ (.) rien ne s'était passé/ (.) alors qu'il venait/ (.) de m'insulter comme jamais j’ai été insultée $\backslash$ (.) jamais/ (.) je me souviens même pas à l'école//(.) on parle du racisme en france/ (.) j'ai jamais/ (.) été insultée comme il l'a dit ${ }^{18}$

Mais si l'échange devient polémique, c'est parce que les propos seront ensuite considérés non seulement comme violents, mais aussi racistes. La confrontation entre ÉZ et HS suscite plusieurs réactions dans les médias, étant considérée comme susceptible de faire réapparaître le spectre du racisme, et comme une diffamation et une incitation à la division au sein de la communauté.

Les propos d'ÉZ sont-ils racistes, ou simplement gênants : que dit la législation audiovisuelle ? Comment définit-elle les notions de racisme et d'incitation à la discrimination et quelles sont les obligations qu'elle impose aux médias audiovisuels en la matière ?

Tout en insistant sur la liberté d'expression, garantie par la loi, étant un droit fondamental, le CSA (Conseil supérieur de l'audiovisuel) précise que cette liberté n'est pas absolue et que « certaines limites s'imposent à son exercice $»{ }^{19}$. Le CSA précise aussi qu'il lutte, entre autres, « contre les discriminations, contre l'incitation à la haine ou à la violence pour des raisons de race, de sexe, de mœurs, de religion ou de nationalité. Le CSA contribue au respect des droits de la personne relatifs à sa vie privée, son image, son honneur et sa réputation, tels qu'ils sont définis par la loi et la jurisprudence, ce qui interdit notamment l'injure et la diffamation $»^{20}$.

Il faut également préciser que le CSA «n’est pas un organe de censure » et qu'il « exerce toujours un contrôle a posteriori, c'est-à-dire

18 « Hapsatou Sy insultée par Éric Zemmour », HapsatouSy, 18/09/2018, 5min08s. https://www.youtube.com/watch? $v=w I B B 231 J K 3 o$ (dernière consultation le 30/01/2021). Nouveau lien : https://www.dailymotion.com/video/x6u113.

19 https://www.csa.fr/Proteger/Éducation-aux-medias-et-a-1-information-EMI/Ressources-pedagogiques/Module-3-Liberte-d-expression-et-ses-limites. Les termes soulignés le sont dans le texte original.

$20 \mathrm{Ibid}$. 
après la diffusion d'un programme [...]. [II] ne peut donc pas intervenir avant la diffusion d'un programme, conformément au principe de liberté de communication $»^{21}$.

D'un point de vue pénal, et selon la législation audiovisuelle, le débat n'a pas à avoir lieu, puisque la séquence n'était pas diffusée. Et si la séquence n'était pas diffusée, c'est parce que les producteurs ont jugé que les propos tombent sous le coup de la $\mathrm{loi}^{22}$. Mais ce qui relève du pénal, relève-t-il de l'éthique ? Ce qui ne peut pas être diffusé, peut-il être dit? Revenant sur l'échange polémique entre ÉZ et HS, Benjamin Castaldi (modérateur de l'émission Touche pas à mon poste!), formule ainsi son point de vue sur la question et sur l'échange polémique entre ÉZ et HS :

4.

BenJAmin CASTALDi on pense pour- à la majorité que ce qui était dit/ (.) euh par zemmour dans l'émission\est absolument/ (.) ABominable $[\ldots]$ dans l'émission ou pas $[\ldots]$ en ce qui me concerne/ je trouve que/ (.) il y a des choses qui ne se disent pas/ (.) et dans ce cas précis/(.) celle-là/ (.) cette phrase-là/ (.) pour moi elle ne devait pas se dire parce qu'elle est absolument/ (.) IMMonde $\backslash$

VoIx c'est pour ça qu'elles ont été coupées//

BENJAMIN CASTALDI mais bon/ elles ont été dites/ dans ce cas-là on dit n'importe quoi/ on coupe/ (.) c'est quand-même dit ${ }^{23}$

Nous pouvons d'abord constater le recours aux adjectifs abominable et immonde (précédés tous les deux par l'adverbe absolument) pour décrire les propos d'ÉZ. Ces adjectifs véhiculent un jugement de valeur négatif sur le contenu propositionnel de l'ensemble du discours

$21 \mathrm{https} / /$ www.csa.fr/Cles-de-1-audiovisuel/Connaitre/Liberte-des-medias-audiovisuels-et-regulation/La-regulation-audiovisuelle-par-le-CSA-avant-ou-apres-la-diffusion.

22 « Thierry Ardisson aurait-il dû défendre Hapsatou Sy ? le producteur Stéphane Simon s'exprime ! », Touche pas à mon poste !, 25/09/2018, 15min28s. https:// www.youtube.com/watch? $\mathrm{v}=\mathrm{dHz} 09 \mathrm{r}-\mathrm{oDAg}$.

23 Ibid. 
de l'invité. Il y a, également et surtout vers la fin de l'extrait ‘4, un questionnement sous-jacent portant sur la responsabilité des producteurs : Peut-on autoriser que ce genre de discours soit même formulé ? Si la réponse du locuteur (Castaldi) est univoque et explicite (ce qui ne peut pas être diffusé ne peut pas être dit), la question mérite toutefois qu'on s'y arrête.

En effet, tout au long de la confrontation (dans ses deux versions, sans et avec montage), TA n'intervient pas dans la polémique, ce qui pourrait être vu comme logique, étant donné son rôle de modérateur. Contrairement aux chroniqueurs, il n'est pas censé participer aux débats. Comme le précise Pérennec (2001, p. 21), le modérateur participe « en amont du débat » et assume « les séquences d'ouverture et de clôture, face caméra ». En tant que présentateur, modérateur de la parole, TA adopte la stratégie d'effacement énonciatif. Il est censé être distant par rapport aux échanges. Il n'est pas non plus censé donner son propre avis sur la question. Mais la responsabilité énonciative rime-telle toujours avec la responsabilité éthique?

Tout producteur doit certainement garantir à son invité la liberté d'expression. Néanmoins, le fait de laisser passer, sans les commenter, sans même s'y arrêter, des propos jugés, par les producteurs euxmêmes, comme diffamatoires et choquants ${ }^{24}$, n'est-il pas une forme de tolérance vis-à-vis de ce discours ${ }^{25}$ ?

La non-intervention de TA n'a pas été toujours interprétée comme une forme d'impartialité ou de professionnalisme : certains ont reproché à l'animateur/modérateur des Terriens de ne pas avoir soutenu sa chroniqueuse dans son désarroi, voire de ne pas avoir défendu certaines valeurs et principes ${ }^{26}$. En choisissant la posture à suivre dans la modération du débat, à savoir celle d'effacement énonciatif, en décidant de ne pas prendre position, TA prend, paradoxalement, une position. Comme le constate Rabatel (2017, p. 131), faire écho à certaines manières de

24 Le producteur Stéphane Simon qualifie les propos d’ÉZ de « choquants » et les catégorise comme des « propos contre la loi » et « qui pouvaient être considérés comme des incitations à la discrimination », " Thierry Ardisson aurait-il dû défendre Hapsatou Sy? le producteur Stéphane Simon s'exprime !", Touche pas à mon poste !, 25/09/2018, 15min28s. https:/www.youtube.com/watch?v=dHz09r-oDAg.

25 Voir, sur ce sujet, Rabatel et Chauvin-Vileno (2006) ; Rabatel et Monte (2017) ou encore Koren (2004).

26 « Thierry Ardisson aurait-il dû défendre Hapsatou Sy ? le producteur Stéphane Simon s'exprime ! », Touche pas à mon poste !, 25/09/2018, 15min28s. https:// www.youtube.com/watch?v=dHz09r-oDAg. 
voir « sans s'en distancier, au nom de l'objectivité, ne participe-t-il pas, fût-ce indirectement, de la reconduction de manières de voir et de penser qui mériteraient par ailleurs d'être discutées ?»

\section{Qui a créé le buzz? Choix de la production et sur- énonciation}

L'affaire Zemmour/Sy a été relatée et commentée dans des émissions de différentes natures (émissions de décryptage de l'actualité27, émissions-débats ${ }^{28}$, notamment). Dans les émissions de décryptage de l'actualité, la confrontation ravive (voire fait renaître) le débat sur la francisation des prénoms et le remet à l'avant-scène. À quelques exceptions près, les débats télévisuels traitant de la question des prénoms sont en interaction directe avec ce que les médias ont baptisé le clash Zemmour/Sy et ils s'inscrivent dans le cadre de cette polémique. Dans les émissions-débats, l'intérêt porte surtout sur l'échange en soi et sur les conséquences de cette affaire et ses rebondissements.

Le diagramme suivant permet de visualiser la présence de l'affaire Zemmour/Sy dans différents genres d'émissions :

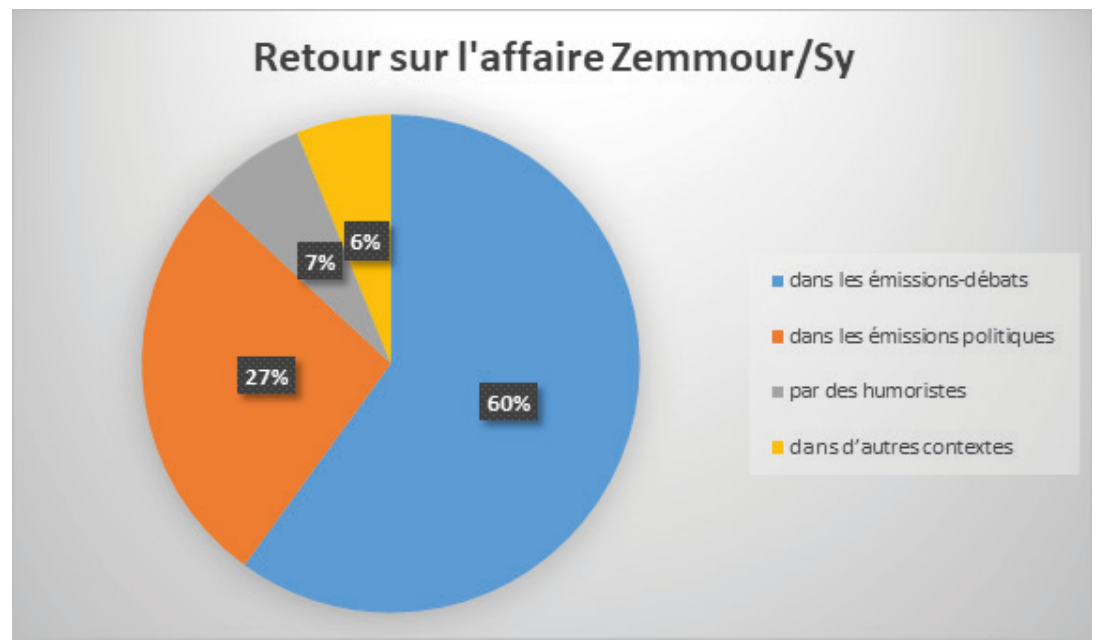

27 Voir, à titre d'exemple, Punchline 17/09/2018, CNews, 17/09/2018, https:// www.youtube.com/watch? $v=R Z A \_I h Q T k Y 8 \& \mathrm{t}=1884 \mathrm{~s}$ (dernière consultation le 15.09.2021).

28 « Hapsatou Sy: "Je demande des excuses à Éric Zemmour" ", Touche pas à mon poste !, 27/09/2018, 23min00s. www.youtube.com/watch?v=uyG9S2xuvyE 
Le nombre total de minutes consacrées à l'affaire Zemmour/Sy et le débat annexe sur les prénoms est d'environ 165 minutes (dans l'ensemble de notre corpus), dont 100 minutes de diffusion réalisées dans le cadre d'émissions-débats. Ce sont d'ailleurs ces émissions qui ont attribué à l'échange les titres de clash et buzz.

Collinet (2017, pp. 46-47) explique que le terme buzz, qui rappelle le bourdonnement des abeilles, n'est pas aussi neuf qu'il n'y paraît. C'est plutôt la notion de buzz médiatique qui semble être un néologisme, ou plutôt une néosémie (apparition de nouveaux sens pour une unité lexicale). Le buzz médiatique peut d'abord désigner le fait de créer une rumeur (Be Diaf, 2014, p. 196), de la nourrir et de renforcer le capital symbolique de ses acteurs (Collinet, 2017, p. 47). Le buzz désigne également «l'engouement soudain et éphémère pour ce type de nouvelles » par les journalistes et les commentateurs qui rebondissent sur l'événement (Collinet, 2017, p. 47).

Comme le buzz, le clash est une onomatopée qui « évoque le bruit d'une collision, notamment le choc d'armes métalliques » (Collinet, 2017, p. 47). Le clash médiatique se caractérise notamment par sa violence (que crée la métaphore des armes ${ }^{29}$ ).

Les locuteurs qui ont rebondi sur l'affaire ont chacun leur avis sur la question. Il semble néanmoins y avoir un accord implicite sur un point : il y a une intentionnelle recherche du buzz, mais par qui ?

En revenant sur l'affaire, plusieurs discours mettent l'accent sur le choix de l'invité, qui est au cœur de la polémique. Le nom de Zemmour donne matière à un faisceau de prédications qui ne sont pas neutres dans la construction du discours à venir : inviter ÉZ pour parler des prénoms étrangers devant des chroniqueurs qui s'appellent William, Monia, Natacha, Raquel et Hapsatou, ça risque de mal tourner. La connaissance encyclopédique des auditeurs associe à l'invité une image bien déterminée. En effet, plusieurs commentateurs font une lecture sémiotique et idéologique de la situation dans laquelle le discours a été proféré et formulent ces sous-entendus d'une façon plus ou moins humoristique (dépendant du contexte de l'énonciation). Nous nous contentons d'un seul exemple tiré de notre corpus. Les deux humoristes Odah et Dako reviennent sur l'affaire et la commentent ainsi :

29 Pour le lien métaphorique entre médias et armes/guerre, voir, entre autres, Huyghe (1998). 
5.

ODAH tout le monde a vu un petit peu ce qui s'est passé entre:/ (.) hapsatou sy/ (.) et : éric zemmour//

DAKO nous- nous en fait c'est même pas le fait qu'il ait des propos douteux qui nous a choqués/

ODAH parce que/ éric zemmour qui tient des propos douteux// ((spectateurs rires)) / on est dans le domaine de la vérité générale〉

DAKO oui : il y a des : il y a des choses universelles que tout le monde sait hein/ (.) la terre est ronde/ le ciel est bleu :/ trafic interrompu sur la ligne treize/

ODAH il y a des bases/ ((rires)) non ce qui nous dérange/ (.) c'est/(.) euh le jeu des médias qui consiste à continuer à l'inviter/ et lever les bras au ciel dès qu'il dit une dinguerie

DAKO parce que je suis désolé là ils ont juste regroupé tous les ingrédients/ pour faire une polémique//

ODAH attends/ (.) tu donnes à éric zemmour/ (.) des caméras/ (.) un micro/ un public/ une chroniqueuse qui s'appelle hapsatou/ (.) hé tu viens de réunir les dragon balls/ ((rires)) hein c'est normal qu'il y ait un dragon qui sort $/{ }^{30}$

Les locuteurs évoquent certes le caractère polémique des propos d'ÉZ et le présentent même comme une évidence (on est dans le domaine de la vérité générale). Mais ce posé ne semble pas être le topic de l'énoncé. L'idée centrale véhiculée dans 5 est que les médias conçoivent des scénarios d'affrontement pour ensuite crier au scandale. Les implicites qui découlent de ce posé sont notamment : 1. le clash est artificiellement créé (ils ont juste regroupé tous les ingrédients pour faire une polémique) 2. il devient habituel (à continuer à l'inviter) 3. il n'a rien d'insolite ou d'inattendu (c'est normal qu'il y ait un dragon qui sort).

L'extrait met en effet sur scène ce que les locuteurs ont appelé le jeu des médias : on conçoit des situations d'affrontement, pour avoir de l'audience. Et pour réaliser de telles situations, on invite des personnalités qui suscitent la polémique. Un clash crée une émission passionnante,

30 « Éric Zemmour et les prénoms », Odah_Dako, 23/09/2018, 2min10s. https://www. youtube.com/watch?v=z2MSuyv-W_8. 
croustillante, bref un petit scandale ; alors qu'un débat sérieux sur les prénoms ou sur un essai autobiographique serait plat et ennuyeux.

Il semble néanmoins que ce n'est pas seulement la combinaison invité/chroniqueurs qui est problématique, mais le concept même de l'émission et la combinaison nature de l'émission/thème/invitélintervieweurs. Cette combinaison, délicate, a fait l'objet d'études faites dans d'autres cadres que linguistiques.

Dans une étude statistique consacrée aux personnalités invitées dans des émissions de talk-show et de divertissement diffusées par des chaînes de télévision et de radio françaises sur la période du $1^{\text {er }}$ janvier 2010 au 31 décembre 2015, Luce et Malherbe (2017, en ligne) précisent que les invités sont « issus majoritairement des métiers du cinéma et de la musique ». Cette forte prédominance des métiers du spectacle fait des émissions du talk-show principalement des émissions de divertissement. L'étude définit le talk-show « comme un programme de divertissement de plateau, qui se différencie de l'entretien/interview, du magazine et du débat $»$.

Or, Les Terriens du dimanche semble être un amalgame de divertissement, d'interview et de débat politique. En effet, l'émission est présentée comme du talk-show, mais les questions posées et les sujets des débats, portant sur l'actualité, touchent différents secteurs de la vie publique (politique et sociale). On est déjà devant un premier contraste : gravité des débats dans un cadre de divertissement. Le cadre précisément (décor, chroniqueurs blagueurs et public souvent riant,...), contribue à donner un air de légèreté au contenu de l'émission. Mettre ces questions polémiques et potentiellement sensibles dans un cadre de divertissement pourrait s'avérer un jeu éthiquement dangereux qui vise, surtout, à accroître l'audience, mais à quel prix ? Comme le précise Hekmat $(2020, \S 4)$ :

[1']orientation [du talk-show] vers la polémique [...] n'est pas uniquement au service d'une logique d'information, elle sert aussi une logique de captation, dont la dérive possible vers le sensationnalisme est régulièrement critiquée.

L'hybridité des genres n'est pas propre à l'émission des Terriens. Elle semble inhérente à la catégorie même du talk-show, dont l'objectif 
est de véhiculer une information dans un cadre de divertissement ${ }^{31}$. L'inclusion de l'information dans le divertissement a créé ce qu'on a appelé l'infodivertissement ${ }^{32}$. Laberge (2014; en ligne) définit l'infodivertissement comme un genre " hybride [qui] combine deux genres télévisuels distincts et permet de légitimer ce qui demeure une formule axée essentiellement sur le divertissement en y incluant occasionnellement et à petites doses des touches d'information et de sujets sérieux ».

C'est dans ce choix de l'invité, et eu égard au contexte de l'énonciation, que TA pourrait être considéré comme le sur-énonciateur responsable de la création de la polémique. En effet, l'instance de la production (incarnée dans les personnes de TA et Stéphane Simon), de par son identité, ses pouvoirs et ses droits, joue le rôle de ce que Rabatel (2009; 2017 (chap. 4, 5 et 6)) appelle un sur-énonciateur. C'est une instance surplombante qui, en choisissant les « ingrédients » de l'émission, conçoit et crée les polémiques et les buzz.

\section{Nous présenter le meilleur des mondes : le montage et les coulisses de l'enregistrement}

Dans une intervention par téléphone accordée à la chaîne CNews, TA répond aux questions que pose Jean-Marc Morandini sur l'échange polémique et ses coulisses ${ }^{33}$. TA présente son point de vue personnel, aussi bien que celui de la production, voire de la chaîne. Il tient une position de surplomb en revenant sur les faits : il expose les arguments défendant ses choix en tant que producteur et s'attaquant à HS, l'accusant d'avoir déclenché « le bad buzz», alors qu'elle connaissait bien ÉZ (zemmour on connait zemmour ( $3 \mathrm{~min} 15 \mathrm{~s}$.), hapsatou connait très bien zemmour (3min26s)).

En relatant le contenu de l'histoire, TA en minimise les enjeux (c'est pas la peine de fantasmer (sur le contenu de la séquence coupée)

31 Ces commentaires portent sur le texte analysé et ne peuvent pas être généralisés sur le genre d'infodivertissement. Il n'est en effet pas de notre rôle, ni de nos compétences de disqualifier le genre, ou dans le sens opposé, de le légitimer.

32 Pour une étude plus large sur le genre d'infodivertissement, ses caractéristiques et les différentes polémiques qu'ils suscite, voir l'étude élaborée faite par Bastien (2013).

33 « Thierry Ardisson agacé par Hapsatou Sy : il parle », Actu Médias, 18/09/2018, $6 \mathrm{~min} 21 \mathrm{~s}$. https://www.youtube.com/watch? $\mathrm{v}=\mathrm{y} 99 \mathrm{bkK} 4 \mathrm{~s} 2 \mathrm{Og} \& \mathrm{t}=50 \mathrm{~s} \quad$ (dernière consultation le 30/01/2021). Nouveau lien: https://www.lexpress.fr/actualite/medias/zemmour-chez-ardisson-hapsatou-sy-sur-la-sellette_2035701.html. 
5min50s) mais, en même temps, maintient vigilance et probité (moi je peux pas vous répéter les mêmes propos parce que de toute façon ils tombent sous le coup de la loi// (3min10s)). Vers la fin de l'interview, TA reformule, malgré tout, ces propos, en les attribuant à un énonciateur second (ÉZ) qui enfreint les normes juridiques et légales; alors que lui, l'énonciateur premier, se pose comme respectueux de ces normes.

Dans ce retour sur l'histoire, TA adopte, encore une fois, la posture énonciative de sur-énonciation, comme on assiste à « la coproduction d'un PDV surplombant de L1/E1 qui reformule le PDV en paraissant dire la même chose tout en modifiant à son profit le domaine de pertinence du contenu ou son orientation argumentative » (Rabatel, 2012, p. 35).

Si TA, dans son rôle de " narrateur/reformulateur » de l'histoire, peut être qualifié de sur-énonciateur, c'est parce qu'il sélectionne et réoriente les propos rapportés, tout en clarifiant les positions et les rôles de chacun des énonciateurs seconds, à savoir ÉZ et HS.

TA revient aussi sur la décision de la chaîne de couper la séquence gênante du clash. En prenant cette décision, les producteurs $n$ 'ont fait que leur travail ${ }^{34}$; une affirmation qui sera ensuite reprise par Stéphane Simon $^{35}$ et même par la chroniqueuse Monia Kashmire ${ }^{36}$ qui prend la défense de TA. Si la séquence n'a pas été diffusée, ce n'est pas parce qu'elle heurte la sensibilité d'HS ou des téléspectateurs, mais c'est plutôt pour faire éviter à la chaîne et à ÉZ un procès, puisqu'on ne peut pas porter plainte pour diffamation en se référant à des propos qui «n'ont même pas été diffusés $»^{37}$. Or, qu'en est-il de la responsabilité morale ? Rabatel et Chauvin-Vileno (2006, en ligne) précisent que

34 « Thierry Ardisson agacé par Hapsatou Sy : il parle », Actu Médias, 18/09/2018, $6 \mathrm{~min} 21 \mathrm{~s}$. https: $/ / \mathrm{www}$.youtube $. \mathrm{com} / \mathrm{watch} ? \mathrm{v}=\mathrm{y} 99 \mathrm{bkK} 4 \mathrm{~s} 2 \mathrm{Og} \& \mathrm{t}=50 \mathrm{~s} \quad$ (dernière consultation le 30/01/2021). Nouveau lien: https://www.lexpress.fr/actualite/medias/zemmour-chez-ardisson-hapsatou-sy-sur-la-sellette_2035701.html.

35 « Thierry Ardisson aurait-il dû défendre Hapsatou Sy ? le producteur Stéphane Simon s'exprime ! ", Touche pas à mon poste !, 25/09/2018, 15min28s. https:// www.youtube.com/watch? $\mathrm{v}=\mathrm{dHz} 09 \mathrm{r}-\mathrm{oDAg}$.

36 « Retour sur l'affaire Hapsatou Sy - Éric Zemmour - Les Terriens du Dimanche - 22/09/2018 », Les Terriens, 02/10/2018, 6min29s. https://www.youtube.com/ watch? $\mathrm{v}=\mathrm{cg} 6 \mathrm{Ir} 9 \mathrm{KTO} 2 \mathrm{U} \& \mathrm{t}=1 \mathrm{~s}$.

37 « Menacé de plainte, Éric Zemmour dénonce "le règne de l'émotion et de la victimisation" ", BFMTV, 17/09/2018, 4min55s. https://www.youtube.com/ watch? $=87 \mathrm{xggNQN4zg}$. 
Si la responsabilité pénale obéit à des conditions juridiques très strictes, variables selon les systèmes juridiques, il n'en va pas de même pour la responsabilité éthique, valable en tous temps, en tous lieux et en toutes occasions. C'est pourquoi il est possible de commettre des actions moralement répréhensibles qui échappent à la responsabilité pénale : il y faut simplement de la technique, une bonne connaissance des lois et une certaine rouerie...

La séquence sur la question des prénoms, citée en 1 (séquence avec montage), se clôt par une blague de Monia Kashmire (chroniqueuse), suivie par les rires du public et un grand sourire de TA. Durant la séquence, la bonne humeur semble régner. TA passe ensuite à une nouvelle question et HS continue de faire partie de l'émission.

Cette joyeuse atmosphère que crée le montage pourrait être interprétée comme une forme de complicité et d'entente. Le sourire/rire de TA qui clôt la séquence peut aussi être vu comme une manière de montrer sinon son accord, au moins l'absence de désaccord ou de refus du PDV d'ÉZ. Les signes para-verbaux font aussi partie de la dynamique communicationnelle et participent à la co-construction d'un point de vue commun. ÉZ se pose ainsi en sur-énonciateur, dont le point de vue, surplombant, s'impose.

C'est d'ailleurs pour réfuter son éventuelle complicité qu'HS affirme avoir décidé de diffuser la suite de l'échange. Comme elle le précise sur le plateau de Cyril Hanouna, elle voulait faire savoir qu'elle « ne rigolai $[\mathrm{t}]$ pas du tout à ce moment-là $»^{38}$. Le montage pourrait en effet suggérer et imposer une représentation de la réalité qui ne correspond pas exactement à la représentation que les acteurs ont de la même réalité et encore moins à la réalité elle-même.

Une semaine après la diffusion de l'émission du 16 septembre, l'équipe des Terriens revient sur l'affaire. TA défend, encore une fois, les choix éditoriaux de la production. Les chroniqueurs, eux, expriment leurs émotions et leurs réflexions sur la question.

Le retour des chroniqueurs sur l'affaire Zemmour/Sy lors de l'émission des Terriens du 22/09/2018 révèlent quelques-unes des logiques du marché qui régissent les médias (amplement analysées par Halimi, 2005). Ainsi, par exemple, les différents membres de l'équipe

38 « Hapsatou Sy : "Je demande des excuses à Éric Zemmour" ", Touche pas à mon poste !, 27/09/2018, 23min00s. www.youtube.com/watch?v=uyG9S2xuvyE 
reviennent, a posteriori, sur la personnalité et le parcours de l'invité, employant un double langage qui rend légitimes les questionnements "concernant les critères de choix des invités évoqués plus haut. Lisons les extraits suivants :

6.

TA

éric zemmour c'est pas n'importe qui/ son dernier livre s'est vendu à CINq-cent-mille exemplaires// [...] qu'est-ce que c'est que cette histoire// (.) où maintenant on doit inviter que les gens avec qui on est d'accord//39

7.

Raquel Garrido (chroniqueuse)

on a beau annoncer/ (.) que :: zemmour/ et globalement l'extrême droite a comme/ (.) objectif politique de diviser la société française et de/ (.) nous pousser tous vers une guerre civile// et ben/ (.) en fait/ il a réussi/ quoi $($.$) en deux coups$ de cuillère à pot// (.) le gars il a désagrégé notre équipe (.) et ce qu'il-/ ce qu'il a réussi à faire pour/ (.) notre équipe à nous// (.) c'est ce qu'il veut faire : pour toute la france/ et c'est pour ça qu'il est dangereux $\left.\right|^{40}$

Alors que TA insiste sur la nécessité d'accepter l'Autre (ici, l'extrême droite), quelles que soient ses idées et convictions, de l'accueillir et de lui donner la parole, les chroniqueurs, eux, diabolisent ÉZ, en mettant l'accent sur ses dangereuses intentions. L'oscillation, parmi les membres d'une même équipe, entre l'explicite objectivité dans le choix de l'invité, et son extrême inverse, à savoir la très claire et très catégorique prise de parti vis-à-vis du même invité, ne peut en effet qu'être remarquée.

Ajoutons que le discours de Monia Kashmire, chroniqueuse d'origine tunisienne (un détail capital dans le contexte d'énonciation), est

39 « Thierry Ardisson agacé par Hapsatou Sy : il parle », Actu Médias, 18/09/2018, $6 \mathrm{~min} 21 \mathrm{~s}$. https: $/ / \mathrm{www}$. youtube.com $/ \mathrm{watch} ? \mathrm{v}=\mathrm{y} 99 \mathrm{bkK} 4 \mathrm{~s} 2 \mathrm{Og} \& \mathrm{t}=50 \mathrm{~s} \quad$ (dernière consultation le 30/01/2021). Nouveau lien: https://www.lexpress.fr/actualite/medias/zemmour-chez-ardisson-hapsatou-sy-sur-la-sellette_2035701.html.

40 « Retour sur l'affaire Hapsatou Sy - Éric Zemmour - Les Terriens du Dimanche - 22/09/2018 », Les Terriens, 02/10/2018, 6min29s. https://www.youtube.com/ watch? $=\mathrm{v}=\mathrm{cg} 6 \mathrm{Ir} 9 \mathrm{KTO} 2 \mathrm{U} \& \mathrm{t}=1 \mathrm{~s}$. 
d'une ambiguïté pour le moins... perturbante. La chroniqueuse insiste notamment sur le fait que la ligne éditoriale de l'émission n'est pas en concordance avec l'idéologie raciste et que la non-intervention de TA ne signifie ni une absence de compassion et d'empathie envers HS, ni une adoption des idées extrémistes ${ }^{41}$. En essayant d'accabler l'invité, la chroniqueuse finit par adopter un registre dont la ressemblance avec celui du discours raciste est troublante.

Commençons par lire la transcription de quelques extraits du discours tenu par la chroniqueuse :

8.

Monia Kashmire

j'ai beaucoup de compassion/ euh et d'empathie pour :- pour la douleur qu'a pu ressentir : hapsatou [...] on s'est tous/ (.) fait attaquer $\backslash$ (.) euh moi/ (.) je ne supporte pas le fait de- (.) de- de- qu'on- qu' on se fasse tous attaquer de complaisance// (.) je comprends pas comment est-ce qu'on peut dire que :/ (.) vous [s'adressant à TA] êtes raciste/ (.) alors que vous êtes le seul/ le premier à avoir invité tant d'arabes et d- et de noirs à la télévision//

TA (hochant la tête en signe d'accord)

$\mathrm{y}$ a vingt-cinq ans $\backslash$

Monia Kashmire

je supporte pas qu'on me traite de complaisante juste parce que par pudeur/ (.) je n'ai pas dit/ (.) que (.) le soir même/ (.) où euh éric zemmour était là $\backslash$ (.) mais moi aussi je me suis fait insulter [...] mais parce que/ vous avez fait votre taf/ (.) vous avez coupé la scène au montage $\backslash$ (.) le public ne sait pas/ (.) mais ce soir-là/(.) éric zemmour/ (.) m'a dit/ (.) si ma mère avait été là/ (.) elle vous mettrait deux claques et elle vous insultera en arabe/(..) ça veut dire quoi m'insulter en arabe//(.) c'est raciste// mais vous avez fait votre travail// $[\ldots]$

sur bfm y avait en boucle affaire hapsatou sy et m'ont mis en boucle en image//(.) donc faudrait dire à bfmtv/(.) je ne suis pas hapsatou sy /(.) ((sourire)) y a une différence entre une arabe et une noire/

$41 \mathrm{Ibid}$. 
Dans ce discours, qui se veut antiraciste, nous ne pouvons que constater la consolidation de certains schémas classiques des théories racistes.

D'abord, la chroniqueuse présente TA comme « le premier à avoir invité tant d'arabes et de noirs à la télévision ». Il y a une catégorisation des invités en Arabes et Noirs d'une part et, en toute évidence, en Français, d'autre part.

Ensuite, les propos qu'ÉZ avait/aurait adressés à Monia Kashmire rendent plus saillant le dilemme exposé plus haut (Ce qui ne peut pas être diffusé peut-il être dit ?) et révèle (ou confirme) la présence, sur les plateaux de télévision, de différentes formes de racisme et de sexisme (l'image plutôt dévalorisante d'une femme qui se fait agresser, verbalement et physiquement, par une autre femme qui a recours à des codes verbaux et culturels appartenant à une autre ère culturelle). Reproduire ce discours, même pour le condamner, n'est-il pas une façon de le faire circuler et propager, et donc de le banaliser, de le rendre plus familier à l'oreille?

Et, finalement, il est légitime de se demander : C'est quoi la différence entre une Arabe et une Noire que la chaîne BFM TV n'avait pas prise en compte? Sachant que Monia Kashmire parle de sa photo qui revient en boucle, peut-on interpréter cette différence comme une nuance de ... couleur?

Il semble en tout cas que le discours de la chroniqueuse confirme un ancien constat fait par les sociologues et les linguistes (voir, entre autres études, Laurent \& Leclère, 2013 ou encore Rastier, 2006) : il n'y a de registre propre au discours raciste et un autre propre au discours antiraciste. D'où la pertinence de la pensée que les discours qui s'autoproclament antiracistes tombent souvent dans le même piège : la lutte contre le racisme, la prise de position très subjective du côté des groupes qualifiés de racisés pourrait très facilement tourner au racisme.

\section{Conclusion}

L'objectif de cette étude était d'analyser l'articulation entre postures énonciatives et rôles interactionnels du producteur/présentateur de l'émission Les Terriens du dimanche avant, pendant et après l'échange polémique entre ÉZ et HS. La posture énonciative dominante (dans le discours de TA en tant qu'unité discursive et textuelle) est celle de sur-énonciation. 
En tant que producteur, TA est un sur-énonciateur : un rôle qui se voit dans le choix de l'invité, des questions à aborder, dans la contribution du temps consacré à chaque acteur et à chaque sujet..., bref, dans les choix éditoriaux.

La séquence diffusée de l'émission a montré un présentateur modérateur qui, dans l'objectif d'atteindre et de préserver une certaine forme de neutralité, adopte la stratégie d'effacement énonciatif ; une stratégie qui est censée lui épargner les prises de position explicites. Mais dans la non-prise de position, il semble y avoir un accord tacite. C'est en tout cas l'interprétation que le silence et le rire de TA ont acquis par les commentateurs de la séquence. Le fait de clore la séquence sur le visage riant de TA fait de sa réaction un accord masqué, une validation des propos d'ÉZ. L'accord impliqué ici est reconstruit par l'interprétant ; ce qui pourrait transformer l'effacement énonciatif en une forme de sur-énonciation.

Le regard surplombant du sur-énonciateur est finalement perceptible le lendemain de l'éclat de la polémique. Dans une interview téléphonique, TA commente et évalue les PDV des deux protagonistes de l'histoire, ÉZ et HS, en se situant par rapport à eux.

Dans les émissions qui ont commenté l'affaire Zemmour/Sy, la responsabilité de TA est vue et évaluée, non seulement sur le plan des paroles, mais aussi des actes, et non seulement sur le plan juridique, mais aussi éthique. Ainsi, le non-engagement, le manque d'acte (de soutien, de défense et de prise de parti) est considéré comme une forme d'engagement.

Quoi qu'il en soit, un constat se dégage : l'affaire Zemmour/Sy témoigne de ce pouvoir des médias de discuter des questions sérieuses et potentiellement dangereuses en créant des polémiques, surtout par le choix des invités, qui, rassemblés alors qu'ils sont incompatibles, ne peuvent que créer le buzz, le bad buzz.

\section{Références}

Bastien, F. (2013). Tout le monde en regarde! La politique, le journalisme et l'infodivertissement à la télévision québécoise, Québec : Presses de l'Université Laval.

Be Diaf, L. (2014). Journalistes 2.0. Usages et dilemmes des journalistes contemporains. Paris : L'Harmattan.

Charaudeau, P. (2006). Discours journalistique et positionnements énonciatifs. Frontières et dérives. Semen, 22. doi : https://doi.org/10.4000/semen.2793 
Collinet, F. (2017). Faites le buzz! Un vieux mécanisme rhétorique adapté à l'espace médiatique contemporain ? Studia Romanica Posnaniensia, 44/3, 43-58. doi : https://doi.org/10.14746/strop.2017.443.003

Coulmont, B. \& Simon, P. (2019). Quels prénoms les immigrés donnent-ils à leurs enfants en France ?, Population \& Sociétés, 565(4), 1-4. doi : https://doi.org/10.3917/ popsoc.565.0001

Doury, M. (1995). Duel sur la cinq : dialogue ou trilogue ? Dans C. Kerbrat-Orecchioni \& C. Plantin (Éd.). Le trilogue (pp. 224-249). Coll. Linguistique et sémiologie. Lyon : PUL.

Halimi, S. (2005). Les Nouveaux chiens de garde, $2^{\mathrm{e}}$ édition augmentée. Paris : Raisons d'agir.

Hekmat, I. (2020). Postures énonciatives et rôles interactionnels des modérateurs dans les débats télévisés politiques. Corela hors-série, 32. doi : https://doi.org/10.4000/ corela. 12382

Huyghe, F.-B. (1998). L'arme et le médium ou la transmission en trois métaphores. Les cahiers de médiologie, 6, 119-129. doi : https://doi.org/10.3917/cdm.006.0119

Jost, F. \& Pierre, S. (dir.) (2021). Pour une éthique du regard. Télévision, 12. Paris : CNRS éditions.

Koren, R. (2004). Argumentation, enjeux et pratique de l'"engagement neutre" : le cas de l'écriture de presse. Semen, 17. doi : https://doi.org/10.4000/semen.2308

Laberge, Y. (2014). Review of Tout le monde en regarde! La politique, le journalisme et l'infodivertissement à la télévision québécoise, Globe, 17(1), 236-238. doi : https://doi.org/10.7202/1028643ar

Laurent, S. \& Leclère, T.(dir.) (2013). De quelle couleur sont les Blancs. Des " petits Blancs » des colonies au "racisme anti-Blancs ». Coll. Cahiers libres. Paris : La Découverte.

Luce, R. \& Malherbe, C. (2017). Invités des talk-shows et émissions de divertissement : tous les mêmes? Revue des médias. https://larevuedesmedias.ina.fr/invitesdes-talk-shows-et-emissions-de-divertissement-tous-les-memes

Pérennec, M.-H. (2001). Le rôle du modérateur dans les débats télévisés. Dans I. Behr (dir.), Télévision et internet : le parlé et l'écrit (pp. 9-28). Coll. Monde germanophone. Paris : Presses Sorbonne Nouvelle. https://books.openedition.org/psn/6437

Rabatel, A. (2009). Prise en charge et imputation, ou la prise en charge à responsabilité limitée... Langue française, 162, 71-87. doi : https://doi.org/10.3917/lf.162.0071

Rabatel, A. (2012). Positions, positionnements et postures de l'énonciateur, Travaux neuchâtelois de linguistique, 56, 23-42. https://halshs.archives-ouvertes.fr/ halshs-00769273/document

Rabatel, A. (2017). Pour une lecture linguistique et critique des médias. Empathie, éthique, point(s) de vue. Coll. Linguistique et sociolinguistique. Limoges : Lambert-Lucas.

Rabatel, A. \& Chauvin-Vileno, A. (2006). La question de la responsabilité dans l'écriture de presse. Semen, 22, https://journals.openedition.org/semen/2792

Rabatel, A. \& Monte, M. (2017). Pour une analyse énonciative engagée et responsable des discours médiatiques. Mots. Les langages du politique, 113, 117-132. doi : https://doi.org/10.4000/mots.22693

Rastier, F. (2006). Sémiotique des sites racistes. Mots. Les langages du politique, 80, 73-85. doi : https://doi.org/10.4000/mots. 17113 
Safi, M. (2006). Le processus d'intégration des immigrés en France : inégalités et segmentation, Revue française de sociologie, 47(1), 3-48. doi : https://doi.org/10.3917/ rfs. 471.0003

Schnapper, D. (2007). Qu'est-ce que l'intégration? Coll. Folio actuel. Paris : Gallimard.

Servais, Ch. (2013). Énonciation journalistique et espace public. Une hégémonie pleine de voix ?. Communication, 32(2). doi : https://doi.org/10.4000/communication.5058

\section{Sites web}

Conseil Supérieur de l'audiovisuel : https://www.csa.fr

Dailymotion: https://www.dailymotion.com/us

Haut Conseil à l'intégration : http://archives.hci.gouv.fr/-Mots-de-1-integration-.html

L'express: https://www.lexpress.fr/ 


\section{(c) (1) $(9)$}

BY NC ND Publié sous la licence Creative Common

«Attibution - pas d'utilisation Commerciale - Pas de Modification 4.0. International» (CC BY-NC-ND) 